\title{
Time and temperature dependent cyclic plasticity and fatigue crack growth of the nickel-base Alloy617B - experiments and models
}

\author{
Gerhard Maier $^{1, a}$, Hermann Riedel ${ }^{1, b}$, Thomas Seifert ${ }^{1, c}$, Jutta Klöwer ${ }^{2, d}$, \\ Ralf Mohrmann ${ }^{3, e}$ \\ ${ }^{1}$ Fraunhofer Institute for Mechanics of Materials IWM, Wöhlerstaße 11, 79108 Freiburg, Germany \\ ${ }^{2}$ ThyssenKrupp VDM GmbH, ${ }^{3}$ RWE Technology $\mathrm{GmbH}$ \\ agerhard.maier@iwm.fraunhofer.de, bhermann.riedel@iwm.fraunhofer.de,
}

cthomas.seifert@iwm.fraunhofer.de, djutta.kloewer@thyssenkrupp.com, ${ }^{\mathrm{e}}$ ralf.mohrmann@rwe.com

Key words: Alloy617, heat treatment, thermomechanical fatigue, cyclic deformation, fatigue life prediction, crack growth

\begin{abstract}
Isothermal low cycle fatigue and thermomechanical fatigue tests are performed on Alloy617B in the solution-annealed and stabilized condition at temperatures between room temperature and $900{ }^{\circ} \mathrm{C}$. In addition, the replica technique is applied to study the growth of microcracks. The Chaboche model is found to describe the cyclic viscoplastic behavior of both heats, except the pronounced cyclic hardening in the low-temperature branches of the TMF tests. A lifetime model based on the cyclic crack-tip opening displacement and the cyclic $\mathrm{J}$ integral is used to describe the measured lifetimes and crack growth rates. However, the description is not fully consistent, since the data for room temperature and for temperatures above $400{ }^{\circ} \mathrm{C}$ fall into two separate scatter bands.

\section{Introduction}

The nickel-base Alloy617 is a candidate for application in new ultra super critical (USC) power plants with steam inlet temperatures of $700 / 720^{\circ} \mathrm{C}$ and pressure of 350 bar with the aim to improve thermal efficiency of fossil fuel power plants and therefore reduce $\mathrm{CO}_{2}$ emission. To meet the creep strength requirements of the key components of the USC power plants, a modified version of Alloy617, Alloy617B, with improved creep strength in the temperature range from 650 to $720{ }^{\circ} \mathrm{C}$ has been developed [1,2]. In addition to creep exposure, the material undergoes transient temperatures during start-up and shut-down. Especially in thick-walled structures, the transients result in temperature gradients and constrained thermal strains that may locally induce stresses above the yield strength of the material. Thus, beside the long-term creep strength, the thermomechanical fatigue (TMF) resistance of the material plays an important role. Furthermore, the material is known to be susceptible to relaxation cracking during service. It is believed that the major cause of relaxation cracking is loss of high temperature ductility due to age hardening at service temperature [3]. To reduce the susceptibility to relaxation cracking a stabilizing heat treatment was introduced, whereas the alloy is conventionally used in the solution-annealed condition [4]. It is the aim of this work to investigate the isothermal low cycle fatigue (LCF) and TMF properties of Alloy617B in the solution-annealed and stabilized material condition and to derive suitable material models for the description of time and temperature dependent cyclic plasticity and fatigue crack growth behavior of Alloy617B.
\end{abstract}

\section{Material and Experimental}

Alloy617B (NiCr23Co12Mo B, Nicrofer ${ }^{\circledR} 5520 \mathrm{CoB}$ ) is a special version of the conventional Alloy617 with narrower limits for selected elements. The chemical composition is $22.2 \mathrm{Cr}, 11.6 \mathrm{Co}$, 8.7 Mo, 1.0 Al, 0.06 C, 1.0 Fe, 0.36 Ti, 0.14 Si, 0.06 Mn, 0.004 B, balance Ni (all quantities in wt $\%)$. 
The material was supplied by ThyssenKrupp VDM in the form of $30 \mathrm{~mm}$ thick hot rolled sheets in two different heat treatments, namely solution-annealed (LG, $1175^{\circ} \mathrm{C} / 1 \mathrm{~h} /$ water) and stabilized (SGH, $1175^{\circ} \mathrm{C} / 1 \mathrm{~h} /$ water $+980{ }^{\circ} \mathrm{C} / 3 \mathrm{~h} /$ air). $\mathrm{SGH}$ material shows a higher number of secondary carbides than LG material. From the supplied sheets cylindrical fatigue specimens with $7 \mathrm{~mm}$ gauge diameter and $19 \mathrm{~mm}$ reduced section length were machined. All fatigue tests were strain and temperature controlled. Complex LCF (CLCF) tests were performed at room temperature (RT), $400,600,700,800$ and $900{ }^{\circ} \mathrm{C}$ with strain amplitudes varying from 0.2 to $0.9 \%$. Each CLCF experiment consisted of an aperiodic and periodic part (Fig. 1) [5].

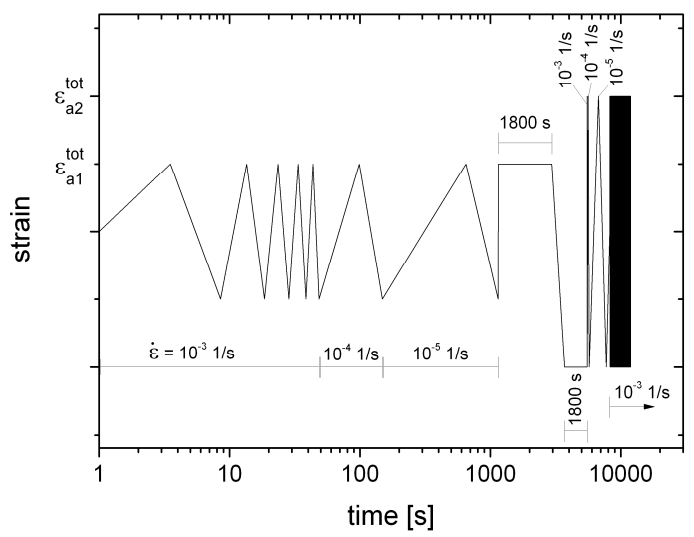

Figure 1: Controlled strain in CLCF test, aperiodic and periodic part (schematic)

In-Phase (IP) and out-of-phase (OP) TMF tests were performed in different temperature ranges. During OP TMF tests, total strain $\varepsilon^{\text {tot }}=\varepsilon^{\text {mech }}+\varepsilon^{\text {th }}$ was kept zero throughout the test. $\varepsilon^{\text {mech }}$ is the mechanical strain and $\varepsilon^{\text {th }}$ the thermal strain. IP TMF tests were performed with $\varepsilon^{\text {tot }}=1.7 \varepsilon^{\text {th }}$. The number of cycles to failure is determined based on a $5 \%$ stress drop of the maximum stress. The temperature dependent fatigue crack growth of individual cracks was measured at RT and $700{ }^{\circ} \mathrm{C}$ in the stabilized condition using the replica technique.

\section{Time and temperature dependent cyclic plasticity of Alloy617B}

\subsection{Experimental Results}

In Fig. 2 the stress response to the aperiodic and periodic part of the CLCF tests is shown for selected strain amplitudes and temperatures. Stress relaxation during hold times in the aperiodic parts is first visible at $700{ }^{\circ} \mathrm{C}$. Pronounced strain rate dependency is observed only at 800 and $900{ }^{\circ} \mathrm{C}$. At RT, both materials, LG and SGH, exhibit a relatively short period of cyclic hardening in the periodic loading stage followed by a period of nearly stable peak stress with minor cyclic softening. Toward the end of the tests, the stress decreases rapidly indicating the formation of macrocracks. In general, at RT the SGH material shows slightly higher peak stresses than the LG material. At higher temperatures the alloy undergoes continuous cyclic hardening with a slightly higher degree of hardening for the LG material than for the SGH material. This effect increases with temperatures up to $800{ }^{\circ} \mathrm{C}$. At $800^{\circ} \mathrm{C}$, the $\mathrm{LG}$ material shows higher stable peak stresses compared to SGH, where cyclic hardening seems to be saturated already after few loading cycles. At $900{ }^{\circ} \mathrm{C}$, there is no difference in material response of the two different heats. Furthermore, for temperatures from 400 to $900{ }^{\circ} \mathrm{C}$, dynamic strain aging is observed by serrated flow in the stress curves. The abrupt stress drops are not shown in Fig. 2.

The results of selected TMF tests are shown in Fig. 3. At the beginning of each test, peak stresses in tension and compression appear to be almost identical. Subsequently, cyclic hardening occurs with a higher stress increase at minimum temperature (OP TMF: tension, IP TMF: compression) than at maximum temperature. Furthermore, in the initial stage of loading, dynamic strain aging was observed during heating for temperatures higher than $550^{\circ} \mathrm{C}$. Again, LG material shows a higher degree of cyclic hardening than the SGH material. 


\subsection{Chaboche Model}

For the description of the experimentally observed material behavior the Chaboche model [6,7] is used, since it is able to describe the essential phenomena of time and temperature dependent cyclic plasticity, namely strain rate dependency, creep, relaxation, recovery as well as the Bauschinger effect and cyclic hardening and softening. The total strain rate of the Chaboche model is given by

$$
\dot{\varepsilon}^{t o t}=\dot{\varepsilon}^{v p}+\dot{\varepsilon}^{t h}+\frac{\dot{\sigma}}{E}-\frac{\partial E}{\partial T} \frac{1}{E^{2}} \dot{T} \sigma
$$

with the viscoplastic strain rate $\dot{\varepsilon}^{v p}$, the thermal strain rate $\dot{\varepsilon}^{\text {th }}$ and the stress rate $\dot{\sigma}$. Young's modulus $E$ depends on temperature. The viscoplastic strain rate has a power law form:

$$
\dot{\varepsilon}^{v p}=\dot{p} \operatorname{sgn}(\sigma-X) \text { with } \dot{p}=\left\langle\frac{|\sigma-X|-\left(y_{0}+R\right)}{K}\right\rangle^{n} \text { and } R=Q_{\infty}(1-\exp [-b p]) .
$$

$X$ is the backstress and $\dot{p}$ the equivalent viscoplastic strain rate. $K$ and $n$ are temperature dependent model parameters. $y_{0}$ is the initial yield stress and $R$ the isotropic hardening function with the temperature dependent material parameters $Q_{\infty}$ and $b$. The backstress tensor is the sum of two parts, $X=X_{1}+X_{2}$, each following the evolution equation [6,7]

$$
\dot{X}_{i}=C_{i} \dot{\varepsilon}^{v p}-\gamma_{i} \varphi_{i} \dot{p} X_{i}-R_{i}^{*} X_{i}+\frac{\partial C_{i}}{\partial T} \frac{1}{C_{i}} \dot{T} X_{i} \text { and } \varphi_{i}=\varphi_{\infty, i}+\left(1-\varphi_{\infty, i}\right) \exp \left[-\varpi_{i} p\right] .
$$

Cyclic hardening and softening is described with the functions $\varphi_{i}$. The temperature dependent model parameters are $C_{i}, \gamma_{i}, R_{i}^{*}, \varphi_{\infty, i}$ and $\omega_{i}$.

\subsection{Adjustment of model parameters}

For the calibration of model parameters, the isothermal CLCF tests are used. The model parameters are determined by a least square fit for all tested temperatures separately. To account for the evolution of the microstructure depending on the preceding heat treatment, the isotropic hardening parameters $Q_{\infty}, b, y_{0}$ are identified for each material condition individually, whereas all other material parameters are the same for both heat treatments. The difference in isotropic hardening behavior can also be observed in the experiments. Fig. 2 shows results of the model adjustment to CLCF tests for selected temperatures. The deformation model is able to describe the time and temperature dependent cyclic material behavior of both heat treatments well.

\subsection{Predictions of the adjusted Chaboche model at non-isothermal loading}

The adjusted Chaboche model was used to predict stresses and strains for non-isothermal loading conditions. In Fig. 3 the model prediction of selected TMF tests are shown for both heat treatments. The temperature dependent model parameters are interpolated linearly in temperature. In general, deformation under non-isothermal loading can be predicted well by the adjusted models, except for the pronounced cyclic hardening observed in the low temperature part of the TMF cycles. For component simulations, the Chaboche model was implemented in ABAQUS/Standard using the implicit algorithms described in $[8,9]$.

\section{Fatigue crack growth of Alloy617B}

\subsection{Mechanism-based lifetime model for LCF and TMF tests}

In LCF and TMF tests with relatively high amplitudes, microcracks are known to nucleate early in the lifetime so that microcrack propagation is the lifetime limiting factor. Here we use the idea that the crack advance per loading cycle, $d a / d N$, is proportional to the crack-tip opening displacement

$$
\frac{d a}{d N}=\beta \Delta C T O D \text {. }
$$



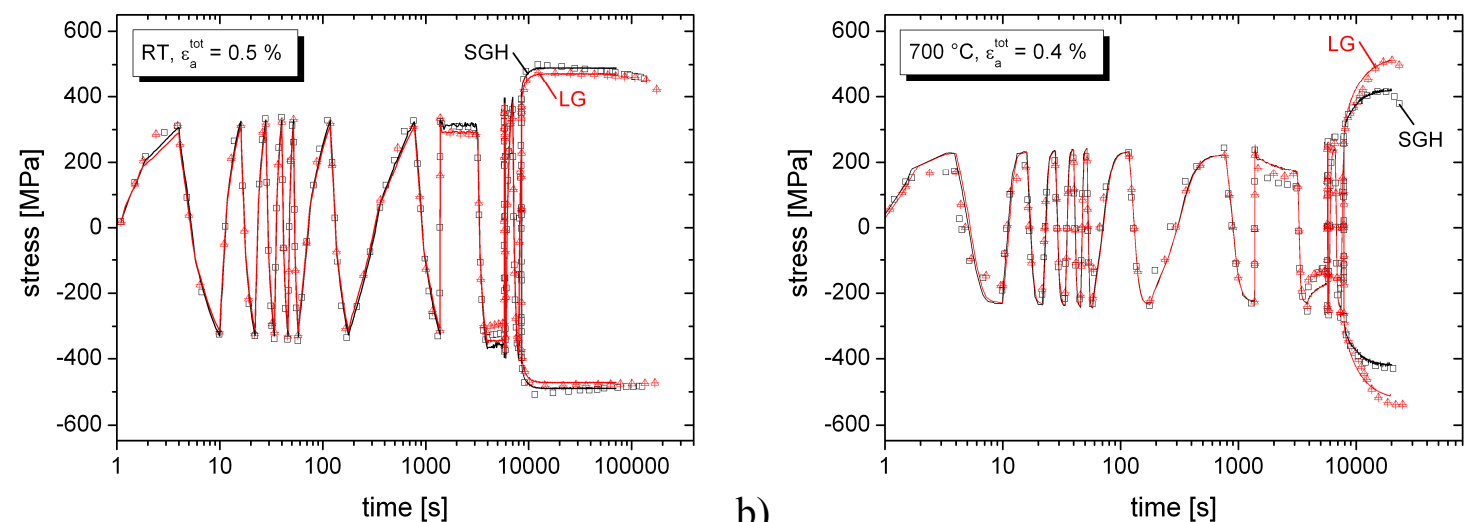

a)

b)
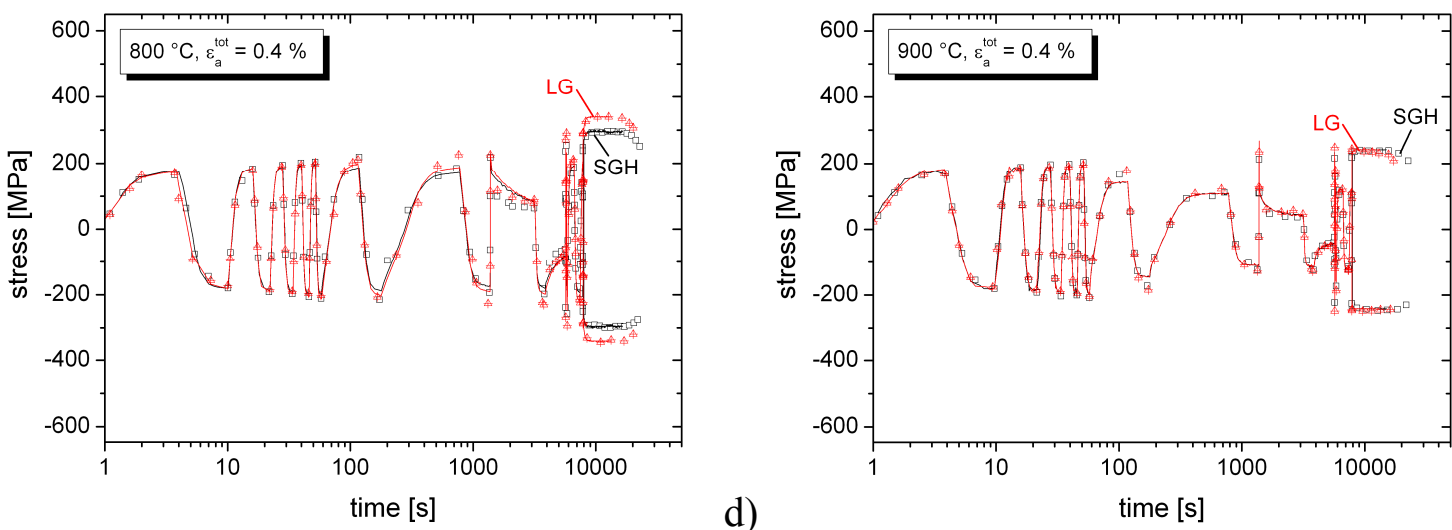

Figure 2: Comparison of experimental data and model prediction of CLCF tests with LG and SGH material. a) RT, $\varepsilon_{a, t}=0.5 \%$, b) $700{ }^{\circ} \mathrm{C}, \varepsilon_{a, t}=0.4 \%$, c) $800{ }^{\circ} \mathrm{C}, \varepsilon_{a, t}=0.4 \%$, d) $900{ }^{\circ} \mathrm{C}, \varepsilon_{a, t}=0.4 \%$.

Symbols represent experimental data, curves the model response. In the periodic part only peak stresses are shown. Experimental data are significantly reduced and are shown till specimen failure.
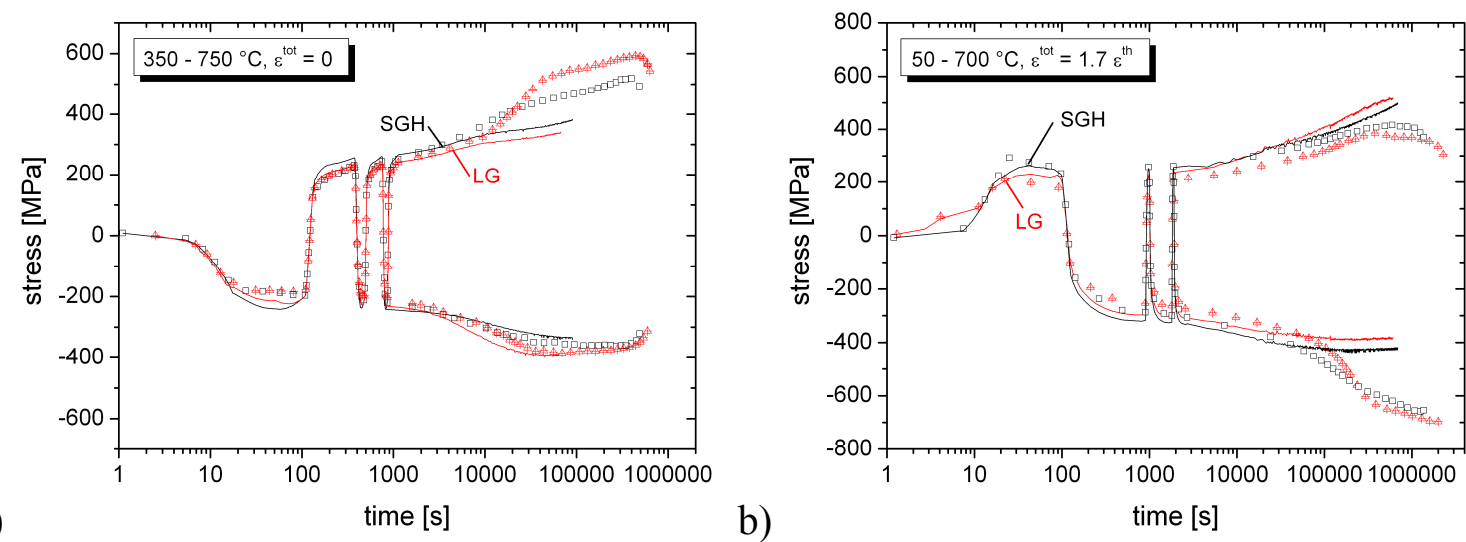

Figure 3: Comparison of experimental data and model prediction of TMF tests with LG and SGH material a) OP TMF, $350-750{ }^{\circ} \mathrm{C}, \varepsilon^{\text {tot }}=0$; b) IP TMF, $50-700{ }^{\circ} \mathrm{C}, \varepsilon^{\text {tot }}=1.7 \varepsilon^{\text {th }}$. Symbols represent experimental data, curves the model response. After three cycles only peak stresses are shown. Experimental data are significantly reduced and are shown till specimen failure. 
The analytical estimate $\triangle C T O D=d_{N} Z_{D} a / \sigma_{c y}$ is used, where $d_{N}$ is a function of the RambergOsgood hardening exponent $\mathrm{N}$ and was tabulated by Shih [10], $\sigma_{c y}$ is the cyclic yield stress, $a$ the crack length and $Z_{D}$ a damage parameter, proposed by Heitmann et al. [11]:

$$
Z_{D}=1.45 \frac{\Delta \sigma_{e f f}^{2}}{E}+\frac{2.4}{\sqrt{1+3 N}} \Delta \sigma \Delta \varepsilon^{p l} \text { with } \Delta \sigma_{e f f}=\frac{\left(1-\sigma_{o p} / \sigma_{\max }\right)}{1-R} \Delta \sigma .
$$

$Z_{D}$ was derived for semicircular surface cracks and rate-independent materials. $E$ is Young's modulus, $\Delta \sigma$ and $\Delta \varepsilon^{p l}$ the stress and plastic strain range. The effective stress range $\Delta \sigma_{e f f}$ according to [12] accounts for the effect of crack closure in the elastic part of $Z_{D}$, where $\sigma_{o p}$ is the crack opening stress, $\sigma_{\max }$ the maximum stress and $R$ the ratio of minimum and maximum stress. By integration of (4), the number of cycles to failure $N_{f}$ can be calculated by

$$
N_{f}=A\left(d_{N} \frac{Z_{D}}{\sigma_{c y}}\right)^{-1} \text { with } A=\frac{\ln \left(a_{f} / a_{0}\right)}{\beta}
$$

with the factor A, including the crack length at failure $a_{f}$ and the initial crack length $a_{0}$.

Fig. 4 shows the correlation between the damage parameter $d_{N} Z_{D} / \sigma_{c y}$ and $N_{f}$ for all LCF and TMF tests. For the calculation of the damage parameter, hysteresis loops at $N_{f} / 2$ are analyzed. The dashed lines in Fig. 4 represent the prediction according to Eq. (6). Apparently the tests with temperatures above $400{ }^{\circ} \mathrm{C}$ fall into a common scatter band around the theoretical line with $\mathrm{A}=6$, whereas the room temperature data lie near a line with $\mathrm{A}=48$, and the $400{ }^{\circ} \mathrm{C}$ lie in between. The stabilizing heat treatment extends the lifetime only at 800 and $900{ }^{\circ} \mathrm{C}$ whereas at lower temperatures LG specimens exhibit longer fatigue lives than SGH specimens.

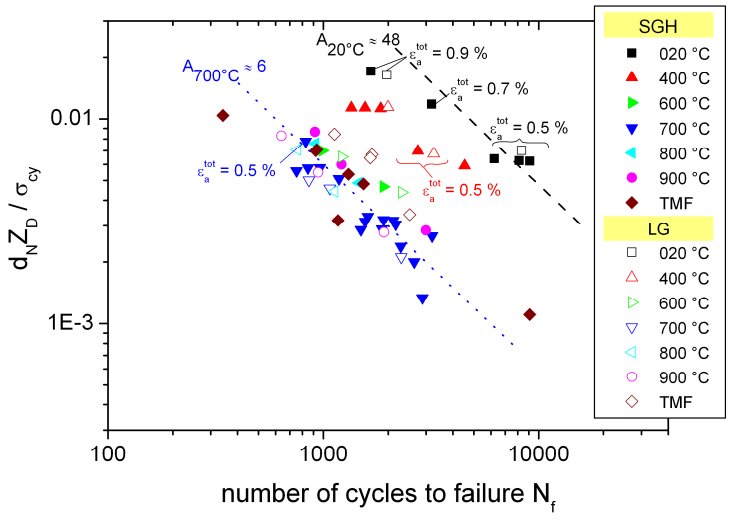

Figure 4: Damage parameter $d_{N} Z_{D} / \sigma_{c y}$ over number of cycles to failure $N_{f}$ for all tests with LG and SGH material.

\subsection{Crack growth behavior of Alloy617B}

In order to understand the difference between the RT and the high temperature lifetimes, the replica technique was used to measure the propagation of microcracks. Two such tests were carried out, one at RT, the other at $700{ }^{\circ} \mathrm{C}$, both with total strain amplitude of $0.5 \%$. Fig. 5 shows the measured crack lengths as a function of the number of cycles $\mathrm{N}$.

a)

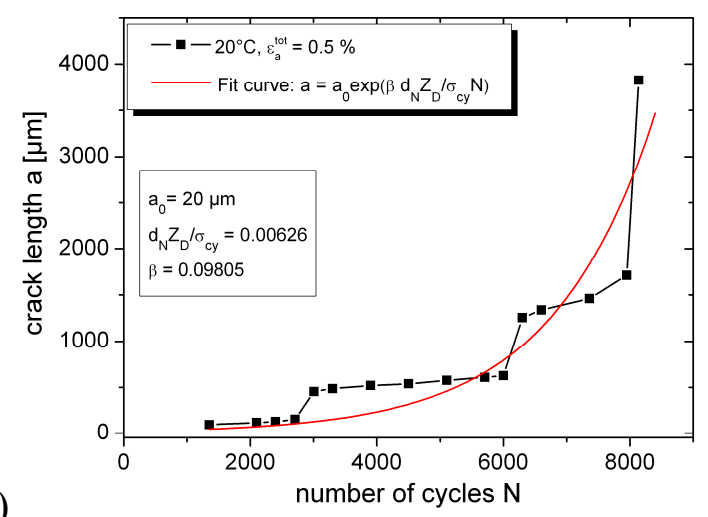

b)

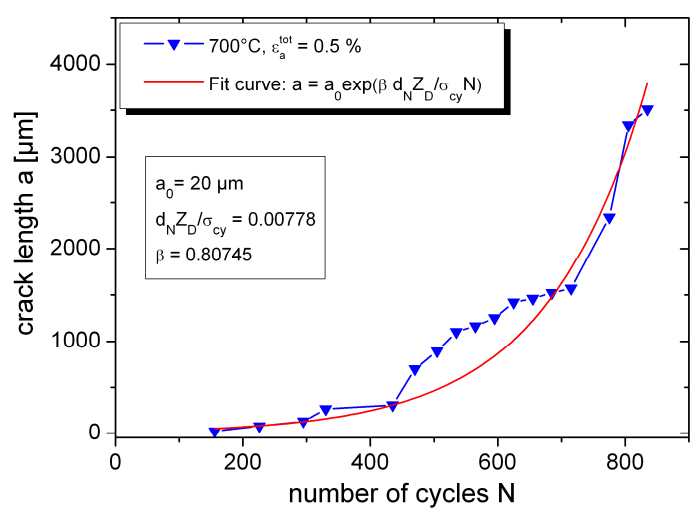

Figure 5: Evolution of the crack length at RT (a) and $700{ }^{\circ} \mathrm{C}$ (b). Lines with symbols: measured at the specimen surface; solid lines: calculated from eq. (4) with adjusted $\beta$. 
The curves are characterized by periods of slow growth interrupted by large jumps, which correspond to crack coalescence events. The jumps are more pronounced at RT (Fig. 5 a) than at $700{ }^{\circ} \mathrm{C}$ (Fig. $5 \mathrm{~b}$ ). This intermittent crack growth is caused by the coalescence of surface microcracks. After coalescence, crack growth at the surface is delayed until the crack has established its approximately semicircular equilibrium shape by preferential growth in depth direction. Hence, despite the seemingly irregular crack extension at the surface, the overall growth rate is controlled by the steady growth in depth direction, and it is therefore justified to fit the theoretical growth law $a=a_{0} \exp \left(\beta d_{N} Z_{D} / \sigma_{c y} N\right)$ derived from eq. (4) to the measured curves. The fit is shown in Fig. 5, with the adjustable parameter $\beta=0.09805$ at $\mathrm{RT}$ and $\beta=0.80745$ at $700{ }^{\circ} \mathrm{C}$, whereas Tvergaard and Hutchinson [13] propose a theoretical value of about $\beta=0.3$. Hence the difference in lifetime by a factor of eight is reflected by the same difference in crack growth rate, but an explanation for this could not yet be found.

\section{Discussion and conclusion}

Viscoplastic deformations measured in the performed thermomechanical and isothermal low cycle fatigue tests depend on the heat treatment and thus on the microstructure. The measured fatigue lifes are almost identical for both material conditions: stabilizing heat treatment extends lifetime in isothermal LCF tests only at 800 and $900{ }^{\circ} \mathrm{C}$. The Chaboche model describes the viscoplastic material response adequately for the tests performed in this work, except for the pronounced cyclic hardening observed in TMF tests. This is probably caused by the evolution of the precipitate microstructure during the tests, which is not taken into account by the model at the present time. Interestingly, the differences between the two heats (solution-annealed vs. stabilized) can be captured by adjusting the isotropic hardening parameters only, rather than the whole parameter set. A lifetime model based on cyclic crack-tip opening and the cyclic $\mathrm{J}$ integral for microcrack growth allows a unified description of the lifetimes in all LCF and TMF tests above $400{ }^{\circ} \mathrm{C}$, while the room temperature data fall into a separate scatter band with about eight times longer lifetimes. Measurements of the crack length evolution by the replica technique yield the result that microcracks grow by about a factor eight faster at high than at low temperature, which is consistent with the measured difference in lifetimes. The lifetime model, on the other hand, would predict a common scatter band for all temperatures. Within this work the reasons for the difference in crack growth rate and lifetime at RT and high temperatures could not be identified.

\section{Acknowledgment}

Financial support of ThyssenKrupp VDM GmbH and RWE Power AG is gratefully acknowledged.

\section{References}

[1] Final report, project no. A130, AVIF, contract no. 0327062, BMWA (MARCKO-DE2), (2005)

[2] R. Blum and R. W. Vanstone, in Materials for Advanced Power Engineering 2006,

Proceedings of the 8th Liege Conference (2006), pp.41.

[3] H. van Wortel, Proceedings of the CORROSION 2007 Conference (2007), Paper No. 07423.

[4] ThyssenKrupp VDM- Werkstoffdatenblatt Nr. 4119, Nicrofer 5520Co - alloy 617 (2005).

[5] T. Seifert, in: M. Borsutzki, S. Geisler (Eds.), Werkstoffprüfung 2006 - Fortschritte der Kennwertermittlung für Forschung und Praxis (2006) 409.

[6] J.L. Chaboche, Int. J. Plast. 2 (1986), 149.

[7] J.L. Chaboche, Int. J. Plast. 5 (1989), 247.

[8] T. Seifert, T. Schenk, I. Schmidt, Comput. Methods Appl. Mech. Engrg. 196 (2007) 2269.

[9] T. Seifert, G. Maier, Int. J. Numer. Meth. Engrg. 75 (2008) 690.

[10] C. F. Shih, J Mech Phys Solids 29 (4) (1981) 305.

[11] H.H. Heitmann, H. Vehoff, P. Neumann, in: S.R. Valluri et al. (Eds.), Advances in Fracture Research 1984 - Proceedings of ICF6, Vol. 5, Pergamon Press, Oxford, New York (1984) 3599.

[12] J. C. Newman, Int. J. Fracture 24 (1984) 131.

[13] V. Tvergaard, J.W. Hutchinson, in: A.F. Blom (Ed.): Fatigue 2002, Proceedings of the 8th International Fatigue Congress held 3 - 7 June 2002, Stockholm, Sweden (2002) 107. 